
Revue
de l'histoire des religions
Revue de l'histoire des religions
$2 \mid 2015$
Les images miraculeuses de la Vierge au premier âge moderne entre dévotion locale et culte universel

\title{
Qui porte le Monde ? Christophe, Atlas, Hercule et Marie (1570-1650)
}

Who Carries the World? Saint Christopher, Atlas, Hercules, and Mary (15701650)

\section{Olivier Christin}

\section{OpenEdition Journals}

Édition électronique

URL : http://journals.openedition.org/rhr/8395

DOI : 10.4000/rhr.8395

ISSN : 2105-2573

Éditeur

Armand Colin

\section{Édition imprimée}

Date de publication : 1 juin 2015

Pagination : 183-210

ISBN : 978-2-200-92965-7

ISSN : 0035-1423

Référence électronique

Olivier Christin, «Qui porte le Monde ? Christophe, Atlas, Hercule et Marie (1570-1650) », Revue de I'histoire des religions [En ligne], 2 | 2015, mis en ligne le 01 juin 2018, consulté le 19 avril 2019. URL : http://journals.openedition.org/rhr/8395 ; DOI : 10.4000/rhr.8395 


\section{Qui porte le Monde? Christophe, Atlas, Hercule et Marie (1570-1650)}

Entre 1570 et 1650, les images qui associent les nouveaux savoirs cosmographiques et l'ancienne cosmologie chrétienne se multiplient. Dans les pages de titre de traités de dévotion, de recueils de cartes des cieux, d'encyclopédies de la Vierge et des saints, notamment, des combinaisons inédites voient le jour, qui montrent Marie, saint Christophe ou saint François prendre la place d'Atlas ou d'Hercule pour soutenir la voute céleste et assurer l'équilibre de l'univers. Ces images composites et savantes témoignent des controverses qui accompagnèrent la diffusion des nouvelles manières de voir et de représenter l'Univers et du travail accompli autour de la Compagnie de Jésus pour apporter une réponse au défi que jetaient les sciences de la nature à la cosmologie de la Renaissance et pour tenter de préserver des cieux christianisés. Cet article examine quelques-unes de ces images et notamment celles de l'Atlas Marianus de Wilhelm Gumppenberg.

\section{Who Carries the World? Saint Christopher, Atlas, Hercules, and Mary (1570-1650)}

Between 1570 and 1650, there was a proliferation of images combining new astronomical knowledge with the older tradition of Christian cosmology. On the title pages of devotional treatises, collections of celestial maps, and encyclopedias of saints' lives, striking new images of Mary, Saint Christopher, and Saint Francis replaced Atlas or Hercules in the role of holding up the celestial vault and assuring the equilibrium of the universe. These composite learned images reveal the controversies accompanying the diffusion of new ways of seeing and representing the universe. They also testify to the efforts of the Society of Jesus to conserve the Christianized heavens in response to the challenge posed by the natural sciences to Renaissance cosmology. This article examines a selection of these images, especially those of the Atlas Marianus by Wilhelm Gumppenberg. 
Mon intention ici est de tenter, de manière sans doute encore très superficielle et donc très provisoire, de recenser et d'expliquer certaines images déroutantes des cieux et de leur organisation qui semblent se multiplier entre 1570 et 1650 et qui ont pour caractéristiques communes de perpétuer quelques principes qui étaient ceux des cosmographies de la fin du Moyen Âge et de la Renaissance (point de vue unifié ou global sur le cosmos, géocentrisme, imbrications des sphères successives), de représenter au cœur de cette conception de l'univers un personnage central emprunté à la mythologie, au récit biblique ou aux vies des saints, comme si ces choix étaient en partie équivalents ou interchangeables et enfin d'associer ce personnage à une vision positive, glorieuse et non pas tragique, du savoir cosmographique évoqué, parfois avec des intentions politiques très explicites. Pour rendre plus clair le type d'images sur lequel je souhaiterais me pencher, mais qui sortent en partie du cadre de ce volume, je peux sans doute me contenter de donner d'emblée quelques exemples, quitte à ne les analyser que de manière trop rapide:

- la première est très connue, puisqu'il s'agit du frontispice de l'édition latine de 1595 de l'Atlas de Mercator paru à Duisbourg' Atlas est ici un géant, ou plus exactement un Titan, que le poids de son châtiment n'écrase nullement. Loin de ployer sous la charge, il paraît tenir sans effort les sphères sur lesquelles il porte un regard fait de curiosité et d'autorité, dans un témoignage assez éloquent d'une confiance nouvelle accordée au savoir. Il est au fond, par certains côtés, figuré en philosophe antique, en sage conscient et certain de son savoir et soucieux d'en faire un usage prudent et raisonné (fig. 1).

- la deuxième image est sans doute un peu moins connue et provient du premier traité de cosmographie publié en anglais, le Cosmographical Glasse de William Cunningham, paru $1559^{2}$ : c'est

1. Gerhard Mercator, Atlas sive Cosmographicae Meditationes de Fabrica Mundi et Fabricati Figura, Duisbourg, 1595. Exemplaire en ligne consultable: http://digital.ub.uni-duesseldorf.de/urn/urn:nbn:de:hbz:061:1-64104.

2. William Cunningham, The Cosmographical Glasse, conteinyng the Pleasant Principles of Cosmographie, Geographie, Hydrographie or Navigation, Londres, John Day, 1559. 
une autre tradition antique que sollicite ici l'auteur de la gravure, qui fait d'Atlas non un Titan puni par les Dieux, mais un roi respecté pour son savoir sur les étoiles, qui porte ici à la fois une couronne et la sphère armillaire construite conformément aux enseignements de Ptolémée et d'Aristote: géocentrisme, emboîtements des différentes sphères toutes figurées comme étant éloignées de la même distance, etc (fig. 2). Cette veine iconographique se retrouve dans des ouvrages d'orfèvrerie plus tardifs, qui tout en renonçant parfois à présenter Atlas comme un roi, n'hésitent pas à le montrer dans toute sa force et sa puissance, triomphant de la charge qui lui a été imposée. C'est le cas, par exemple, d'un travail allemand de la seconde moitié du $\mathrm{XVI}^{\mathrm{e}}$ siècle qui se trouve aujourd'hui conservé à Toulouse à la Fondation Georges Bemberg.

- la troisième est au fond celle qui m'a conduit à aborder ce sujet sur lequel je n'avais jusqu'ici jamais travaillé. Il s'agit d'un tableau daté des années 1570 et conservé aujourd'hui au musée historique de Bâle: un géant, qui traverse un cours d'eau en s'appuyant sur un bâton, porte sur son dos le Christ-Enfant mais aussi une sphère armillaire. Le géant est donc à la fois ou en même temps Atlas et Christophe - et peut-être Hercule. L'étendard du Christ et l'axe de la sphère armillaire ne se confondent pas totalement, mais ils sont assez proches pour entretenir la confusion, et poursuivre le jeu des substitutions ou des équivalences. Christophe portant le Christ porte aussi les cieux; Atlas porte les cieux et le Christ: le spectateur doit comprendre qu'il n'a pas à choisir, que l'image l'incite justement à saisir les deux histoires dans un même regard (fig. 3). Or cette ambiguité n'est au fond pas exceptionnelle: elle se retrouve ailleurs, dans d'autres contextes et dans d'autres supports, à tel point qu'il semble bien qu'il faille la considérer comme structurelle, voulue, propre à un état du champ du savoir et non comme conjoncturelle, fortuite ou curieuse, destinée par exemple à un commanditaire précis, ayant le goût des énigmes et des paradoxes. On peut ici mentionner le frontispisce d'un ouvrage paru en 1704 à Prague, qui représente Atlas, ou en tout cas un géant, portant sur ses épaules un globe où chaque continent est nommé «Maria» ${ }^{3}$ (fig. 4).

3. Antonín Frozín, Obrowisstě Maryánského Atlanta, Prague, Jiří Laboun, 1704. Il s'agit d'une édition partielle de l'Atlas de Gumppenberg. 
- enfin, je m'arrêterai sur une dernière image qui ne prend sens à mon avis que dans le contexte général de celles que je viens d'évoquer. Il s'agit du frontispice d'un ouvrage dont les deux premiers livres paraissent en 1657 en latin ${ }^{4}$ et en allemand ${ }^{5}$, à Ingolstadt et à Munich et qui portent le titre à la fois banal et mystérieux d'Atlas Marianus ${ }^{6}$ (fig. 5). Il s'agit de ce qu'il est aujourd'hui convenu de désigner comme une «topographie sacrée» - recensement et localisation méthodiques de sanctuaires et de pèlerinages - imaginée et patiemment mise en œuvre au cours des années 1650, par un membre de la Compagnie de Jésus, Wilhelm Gumppenberg, qui agissait là en partie comme la plume d'un auteur collectif qui n'était autre que la Compagnie elle-même. La première édition scientifique vient d'en paraître aux éditions Alphil à Neuchâtel sous le titre Marie Mondialisée.

4. L'Atlas Marianus connaît dès ces années de nombreuses éditions: Atlas Marianus sive de Imaginibus Deiparae per Orbem Christianum Miraculosis, auctore Guilielmo Gumppenberg, livres I et II, Ingolstadt, G. Haenlin, 1657, in12, 219 et 207 p. Le premier feuillet porte les mots «editio secundu». Privilège et approbation pour les deux premiers livres du père Servilianus Veihelin, Fribourg, 25 juin 1657; Atlas Marianus sive de Imaginibus Deiparae per Orbem Christianum Miraculosis, livres I et II, Munich, Straub, 1657, in-12, 216 et 207 p.; en tête du livre, on lit «editio tertia»; Atlas Marianus sive de Imaginibus Deiparae per Orbem Christianum Miraculosis, auctore Guilielmo Gumppenberg, livres I et II, Ingolstadt, G. Haenlin, 1657, livre I, 207 p. avec fig., 14 p. pour la Dedication et l'Avis Lectori, livre II, 285 p., index et approbation Fribourg 1657, avec un index generalis pour les quatre livres et un catalogus imaginum, 12 p.; Atlas Marianus sive de Imaginibus Deiparae per Orbem Christianum Miraculosis, auctore Guilielmo Gumppenberg, livre I, frontispice gravé par Küsel, «editio tertia», puis Dedicatio 8 p., Avis lectori 20 p., puis texte 217 p. avec 25 gravures signées GAWG, Munich, Jaecklin, 1657.

5. Marianischer Atlas: das ist wunderthätige Mariabilder so in aller christlichen Welt mit Wunderzaichen berhuembt durch Guilielmum Gumppenberg, livres I et II, Ingolstadt, G. Haenlin, 1657, chacun avec 25 grav.; Marianischer Atlas: das ist wunderthätige Mariabilder so in aller christlichen Welt mit Wunderzaichen berhuembt durch Guilielmum Gumppenberg, livre II, avec texte latin, Munich, Straub, 1657, 207 p., 25 grav.

6. Les livres III et IV, dont la diffusion sera moindre, sont publiés en latin et en allemand en 1659. Une édition critique des livres I à IV accompagnée d'une traduction et de l'ensemble des gravues accompagnant l'ouvrage est parue: Marie Mondialisée L'Atlas Marianus de Wilhelm Gumppenberg, édition et traduction par Olivier Christin, Nicolas Balzamo et Fabrice Flückiger, Neuchâtel, Alphil, 2015. Cf. aussi Olivier Christin, Fabrice Flückiger et Naïma Ghermani (dir.), Marie mondialisée. L'Atlas Marianus de Wilhelm Gumppenberg et les topographies sacrées de l'époque moderne, Neuchâtel, Alphil, 2014. 
L'ouvrage rassemble de courtes notices, conçues toutes sur un même modèle - celui des encyclopédies systématiques et des recueils de lieux communs qui connaissent alors une sorte d'apogée dans les décennies qui précèdent les premières éditions de l'ouvrage de Gumppenberg - et consacrées à cent des plus célèbres sanctuaires mariaux de la Chrétienté européenne, avec de rares extensions vers l'Amérique ibérique et les îles Canaries. Chaque notice, accompagnée d'une gravure, relate ainsi l'histoire de l'image miraculeuse de Marie à l'origine du pèlerinage, en décrit quelques-unes des manifestations miraculeuses décisives, souvent les toutes premières et toujours en tout petit nombre, et s'achève par l'indication très précise des sources et des informations utilisées pour sa rédaction.

Ces premières éditions n'étaient toutefois que l'amorce d'une publication plus vaste, annoncée dès le début des années 1650 dans un petit ouvrage intitulé Idea Atlantis Mariani et achevée en $1672^{7}$. Elle rassemblait cette fois 1200 sanctuaires situés dans le monde entier. Puisque d'une édition à l'autre, on passait de manière spectaculaire d'une géographie régionale ou nationale, d'une topographie pieuse et patriotique à la fois, à une cosmographie universelle, envisageant de décrire la présence de Marie partout dans le monde, la collection, l'organisation et la publication de l'immense somme d'informations rassemblées ne pouvait être envisagée par Wilhelm Gumppenberg qu'à la double condition d'un important travail collaboratif ${ }^{8}$ et du recours à des choix de mise en forme ou de mise en ordre du savoir très particuliers et surtout très révélateurs d'un moment historique et scientifique précis. Étaient ainsi conjugués, dans une architecture sophistiquée, dont témoignent les centaines de pages d'index variés qui multiplient les possibilités de classement, de repérages

7. Wilhelm Gumppenberg, Idea Atlantis Mariani, Trente, Carlo Zanetti, 1655; Wilhelm Gumppenberg, Atlas Marianus, quo sanctae Dei genitricis Mariae imaginum miracolusarum origines duodecim historiarum centuriis explicantur, Munich, Johann Jecklin, 1672.

8. Au Capitulus XIII de l'édition de 1672, notamment dans le premier index (Adjumenta praeterita), les lignes d'introduction précisent que «opus fuit plurium opera», grâce à l'aide des pères de la Compagnie. Il va donc distinguer deux catalogues dans cet index, les pères de la Société qui servirent d'informateurs (catalogus $I$ ) et les historiens ou auteurs sources (catalogus II): le premier comporte 270 noms, le second 177. 
et de croisement ${ }^{9}$, les principes et les méthodes propres aux arts de la mémoire et aux sommes systématiques de lieux communs, d'une part, et les possibilités offertes par le système de l'atlas, d'autre part, forme savante du visuel et forme visuelle du savoir tout à la fois, comme l'a rappelé récemment Georges Didi-Huberman à propos de la vogue au cours de la Renaissance de ce nouvel outil d'appréhension, de rassemblement et de rapprochement de données disparates et sans nombre fini ${ }^{10}$. C'est sur ce point précis que j'aimerais m'arrêter en premier lieu, quitte à laisser de côté provisoirement les autres images que j'évoquais et leurs innombrables variantes entre 1570 à 1650, dans la peinture, le livre scientifique ou la sculpture.

Pour comprendre ce qui se joue dans ce frontispice à première vue étonnant - et dans la page de titre de l'édition finale de 1672 que j'évoquerai plus loin, il faudra sans doute accepter de faire un détour par d'autres images, qui cette fois ne perpétuent pas les présupposés de la cosmologie de la Renaissance. Il s'agira pour l'essentiel de ces pages de titre ou de ces frontispices qui représentent non plus Atlas mais Hercule comme celui qui soulage un moment Atlas dans sa charge pour le laisser cueillir pour lui les pommes du Jardin des Hespérides, conformément à une longue tradition qui remonte à Pausanias et Apollodore. Ce récit mythologique va nourrir de nombreuses images modernes. On peut en donner pour exemple l'Atlas Nouveau de Jaillot (1694) à la gloire du roi de France et de l'Hercule français, le Titan étant entouré de deux statues équestres de Louis XIV et du Dauphin ${ }^{11}$, l'Atlas de Johann Baptist Homann (1710), qui présente Atlas et Hercule dos à dos, conjuguant leurs efforts pour soutenir la voute céleste ${ }^{12}$, ou encore l'Atlas François de Sanson et Jaillot (1700) dont la page de titre montre Atlas venant

9. Un premier index range les sanctuaires non par lieu géographique mais sous la forme de diverses rubriques: la découverte (inventio), les matériaux, la forme, le culte, la nature, les temples, les miracles, puis une table des matières classique qui reproduit l'ordre de présentation des 1200 notices par zones géographiques et encore un index rerum et verborum d'environ 100 pages.

10. Georges Didi-Huberman, Atlas ou le gai savoir inquiet, Paris, Éditions de Minuit, 2011.

11. Alexis-Hubert Jaillot, Atlas Nouveau, contenant toutes les parties du monde..., Paris, Hubert Jaillot, 1694, frontispice.

12. Johann Baptist Homann, Neuer Atlas, bestehend in einig curieusen astronomischen mappen und vielen auserlesenen allerneuesten land-charten über die gantze Welt, Nuremberg, Homann, 1710. 
déposer sur les épaules d'Hercule un globe parsemé de fleurs de lys alors que le sol est jonché des instruments propres au savoir astronomique $^{13}$. D'autres combinaisons sont possibles, comme celle de l'Uranometria de Johann Bayer (1603) qui présente Atlas comme le maitre astronome et Hercule comme son disciple ${ }^{14}$ (fig. 6).

\section{Pourquot un ATLAS ?}

L'Atlas Marianus est l'un des premiers imprimés portant ce titre sans être un recueil de cartes, de portulans, d'isolarii ou de vues et sans comporter la moindre carte ou vue de paysage, à l'exception $\mathrm{du}$ frontispice, sur lequel je reviendrai. Les usages savants du personnage de la mythologie pour désigner une forme d'ouvrage ou de collection de documents à la fois semblables et différents - comme peuvent l'être les plans de diverses villes ou les cartes de plusieurs territoires - sont en effet récents. Ils datent des années 1570, avec les réalisations d'Antonio Lafreri (dans la disposition dite «Italian, Assembled to Order» variant de commanditaire à commanditaire), et pour les volumes reliés de la parution posthume du grand livre de Mercator en 1595 à Duisbourg, avant de devenir extrêmement courants dans les années 1620-1630, grâce à l'activité des imprimeurs des Provinces-Unies au premier rang desquels Hondius et Blaeu. Dans le Saint-Empire, en dépit du précédent de 1595, la nouveauté de ce choix à la fois lexical et méthodologique pour le livre de Gumppenberg devait être frappante, si l'on songe qu'un seul livre publié au $\mathrm{XVI}^{\mathrm{e}}$ siècle portait ce titre (l'Atlas de Mercator) et que sur les 136 titres comportant le mot «atlas» recensés dans le VD $17^{15}$, moins d'une douzaine est antérieure aux premières éditions de Gumppenberg en dehors des publications

13. Alexis-Hubert Jaillot et Nicolas Sanson d'Abbeville, Atlas François, contenant les cartes géographiques dans lesquelles sont très-exactement remarquez les empires, monarchies, royaumes et estats de l'Europe, de l'Asie, de l'Afrique et de l'Amérique, Paris, Hubert Jaillot, 1700.

14. Johann Bayer, Uranometria, omnium asterismorum continens schemata, nova methodo delineata, aeris laminis expressa, Augsbourg, [s. n.], 1603.

15. VD 17. Das Verzeichnis der im deutschen Sprachraum erschienenen Drucke des 17. Jahrhunderts, base de données des imprimés de l'espace germanophone du $\mathrm{XVII}^{\mathrm{e}}$ siècle, établie par la Deutsche Forschungsgemeinschaft (DFG): http://www. vd17.de. 
de Blaeu et Hondius. Il s'agit pour l'essentiel des recensements d'évêques et de territoires épiscopaux, d'un portrait de Sigismond, roi de Pologne, par Lucas Kilian que l'on reverra très actif dans les grands projets de cartes christianisées des cieux, d'un poème et surtout d'un dialogue astrologique de Christoph Richter, un diacre auteur de plusieurs calendriers à partir de 1655 , qui met en scène Atlas, «un astrologue», Bruno, «un astrologue», Corydon, «un paysan», et Daniel, « un bourgeois $»^{16} \ldots$

Même cantonné pour l'essentiel aux ouvrages, assemblés ou non, qui ont pour objet des cartes, des plans, des images de côtes maritimes ou des îles, le personnage connaît alors une carrière fulgurante qui en fait l'une des figures, centrale et incertaine à la fois, de la représentation des sciences de l'homme, c'est-à-dire des sciences que l'homme a forgées et conquises et non de celles qui lui ont été révélées, des sciences qui décrivent de mieux en mieux désormais, grâce à la connaissance et à l'observation directes, le monde qu'il habite et la place qu'il peut prétendre y occuper, des sciences qu'il invente et perfectionne pour se connaître par lui-même. Atlas est ainsi indissolublement symbole et exemple, modèle et admonestation, objet concret et organisation d'un savoir en train de se faire. Chez Andrea Argoli (1610), par exemple, Atlas est présenté face à Hercule comme Vetus Magister d'astronomie ${ }^{17}$; chez Jansonnius au milieu du XVII ${ }^{\mathrm{e}}$ siècle, il est entouré de savants et de sages qui étudient sphères et globes, grâce à lui et grâce à son sacrifice $^{18}$. Ailleurs, Atlas sert de support à des encriers pour des objets destinés à des cabinets de lecture et des bibliothèques et dans l'édition abrégée de Mercator en 1651, Atlas est à la fois un roi et un savant, et en tout cas un roi entouré de savants qui profitent de sa présence, de sa constance sans lesquelles aucun savoir durable ne serait possible.

16. Christoph Richter, Dialogus Astrologicus. Astrologisches Gespräch von dem Zustande des MDCLVIII. Jahres nach der heylsamen Geburt des Herrn Messiae: Die Personen des Gesprächs sind folgende: Atlas, ein Sternseher. Bruno, ein Sternseher. Corydon, ein Bauer. Daniel, ein Bürger. Ernestus, ein Soldat. Florianus, ein Artz, Leipzig, Bauer, [1657]. Nombreuses éditions dans toute la seconde moitié du XVII ${ }^{\mathrm{e}}$ siècle.

17. Andrea Argoli, Tabulae primi mobilis Andreae Argoli: quibus veterum reiectis proliritatibus directiones facillimè componuntur, Rome, Guilelmi Facciotti, 1610.

18. Johann Jansonnius, Novus Atlas sive theatrum orbis terrarium, Amsterdam, Johann Jansonnius, 1649. 
Wilhelm Gumppenberg innove donc en reprenant le nom, mais en refusant la légende qui l'accompagnait et en abandonnant la représentation du personnage de la mythologie. En découvrant la page de titre des premières éditions de l'Atlas Marianus, ses lecteurs ne pouvaient qu'être surpris et peut-être perturbés. Pourquoi choisir ce titre et avec lui un personnage de la mythologie païenne dans lequel on confondait souvent deux figures distinctes - le roi philosophe et mathématicien de Mauritanie qui aurait fabriqué des globes célestes et à qui Mercator fit allusion dans son propre ouvrage ${ }^{19}$; le Titan frère de Prométhée, puni par Zeus et condamné à porter sur ses épaules les cieux ${ }^{20}$ - alors même que ces deux figures et leur confusion pouvaient sembler associer savoir humain et réprobation divine, curiosité intellectuelle et souffrance ${ }^{21}$ ? Pourquoi recourir à Atlas et à la mythologie païenne pour un ouvrage qui se donnait explicitement pour objectif de défendre et de propager le culte marial, érigé en fondement incontestable du monde catholique et de sa représentation particulière du cosmos, et faire ainsi servir à la foi catholique une figure qui lui était a priori étrangère ? Pourquoi procéder à l'inverse de certains atlas hollandais, chez Blaeu par exemple, qui choisissent de christianiser autrement les cieux, en remplaçant Atlas par le Père Éternel, le Tétragramme, ou encore le Christ? Et pourquoi substituer Marie au Titan ou au roi de la mythologie devenus figures emblématiques des nouveaux savoirs cosmographiques et géographiques, en appliquant des ressources rhétoriques et argumentatives revendiquées par les sciences et la

19. Mercator dit lui-même s'être «proposé d'imiter le roi Atlas, aussi remarquable par son érudition et sa bonté que par sa sagesse». Cf. Atlas sive Cosmographicae Meditationes..., Atlantis Pars Altera Authore Gerardo Mercatore: Epistola dedicatoria Rumoldi Mercatoris. «On raconte qu'il était expert en astrologie et qu'il fut le premier à répandre chez les hommes la doctrine de la sphère; c'est pour cette raison que l'on croit que l'univers entier repose sur les épaules d'Atlas, cette légende signifiant la découverte et la description de la sphère» (Diodore de Sicile, Bibliothèque historique, livre III, chap. 60, éd. et trad. par Bibiane Bommelaer, Paris, Les Belles Lettres, vol. III, p. 94).

20. D'après la tradition la plus répandue, Atlas évoque la punition infligée par Zeus après la défaite des Titans (Hésiode, Théogonie, v. 517-520). La tradition qui fait d'Atlas un astronome est plus tardive (voir Virgile, Énéide, livre I, v. 741).

21. La confusion est patente dans le travail d'orfèvre augsbourgeois du $\mathrm{XVI}^{\mathrm{e}}$ siècle aujourd'hui conservé par la fondation Bemberg à Toulouse et cité plus haut: Atlas, le Titan, porte sur ses épaules une immense sphère armillaire, mais avec une facilité qui n'indique en rien la souffrance. Il est une incarnation du savoir triomphant, capable de soulever le monde. 
philosophie de la nature à un propos consacré aux manifestations les plus indicibles et les plus incontestables de la puissance absolue de Dieu (les miracles et les apparitions mariales) au risque de devoir s'obliger à expliquer les équivalences ou les parallèles entre panthéons païen et chrétien, entre formes de savoir sur la nature et connaissance extraordinaire de la surnature, entre raison (ou calcul) et foi ? Comment comprendre, au fond, ce retour, au milieu $\mathrm{du} \mathrm{XVII}^{\mathrm{e}}$ siècle, en plein débat sur la révolution copernicienne et l'utilisation de la lunette pour l'observation directe des planètes et avec eux en pleine remise en cause des conceptions traditionnelles du cosmos, à certaines des ambitions qui furent celles de la cosmographie et de la géographie chrétienne de la fin du Moyen Âge et de l'Humanisme? Gumppenberg livrerait-il un combat perdu depuis longtemps?

Pour répondre en partie à ces questions, qui relèvent à la fois de l'histoire des sciences et de celle des conflits confessionnels, il faut commencer par la page de titre et par les frontispices des premières éditions de Gumppenberg, amplement modifiées par la suite sans que l'étonnement devant cet assemblage de savoirs et de discours disparates ne disparaisse jamais totalement. C'est là en effet que l'on peut tenter de comprendre ce que les choix visuels font aux savoirs scientifiques et religieux et plus précisément dans ce cas, ce que ces «vues de l'esprit »22, qui montrent ce que nul œil humain ne saurait voir, permettent à la pensée de construire et d'imaginer dans la combinaison de faits, d'hypothèses et de spéculations qui englobe les phénomènes terrestres, les espaces sidéraux et les sphères supernaturelles.

Dus à trois graveurs augsbourgeois différents (Mathias ou Melchior Küsel, Wolfgang Kilian, Georg Andreas Wolfgang) et distingués par quelques variantes de détail qui sont sans importance pour notre propos, les frontispices de l'Atlas s'organisent en effet selon un partage de l'espace en trois zones. La partie supérieure dévoile la voûte céleste, où l'on aperçoit le soleil, la lune mais aussi une manière de bande ou de bandeau comportant six figures

22. J'emprunte l'expression à Bruno Latour, «Les 'Vues' de l'Esprit: une introduction à l'anthropologie des sciences et des techniques», Culture technique, numéro spécial, ${ }^{\circ} 14,1985$, p. 5-29, dans un texte qui insiste sur les transformations des $\mathrm{XVI}^{\mathrm{e}}-\mathrm{XVII}^{\mathrm{e}}$ siècles dans la représentation du monde ou plus exactement des «vues du monde». 
sur lesquelles je reviendrai; la partie inférieure montre le globe terrestre et plus précisément un morceau de l'hémisphère nord où sont indiqués les territoires italiens, espagnols, français et ceux de la Germania; entre les deux, se trouve la figure de la Vierge, ou plus précisément une représentation, conforme aux éléments de l'iconographie la plus courante de ce sujet, du transport miraculeux de la Santa Casa de Lorette.

Gumppenberg et ses éditeurs choisissent donc de donner une transcription figurative littérale du titre si étonnant du livre, en combinant deux répertoires visuels bien identifiés: une illustration au fond classique de la Vierge de Lorette et de la Santa Casa, portée dans le Ciel par les anges, et une représentation des sphères célestes et terrestres saisis d'un seul coup d'œil. Le lecteur avait ainsi d'emblée un point de vue global, unique, parfait sur le cosmos qui ne pouvait être que celui de Dieu, dans le droit fil des illustrations de Petrus Apianus au début du XVI ${ }^{\mathrm{e}}$ siècle - où l'on apercevait même un œil saisissant d'un coup la totalité de l'univers et les relations entre ses parties ${ }^{23}$ - et surtout de la page de titre de l'édition de 1595 de Mercator, où Atlas tenait entre ses mains et sous son regard les sphères terrestres et célestes. En ce sens, Marie prend bien ici chez Gumppenberg la place d'Atlas, non dans la souffrance du châtiment, mais en reliant et en séparant tout à la fois le globe terrestre et la voûte céleste et donc en garantissant l'ordre de l'univers et l'harmonie des relations entre ses parties, conformément au projet intellectuel même de la cosmographie de la Renaissance qui se concevait comme le cadre unique de toutes les sciences de la nature. Mais ici, Marie ne supporte pas le fardeau des cieux, ployée sous la charge comme l'Atlas Farnèse dont la posture ressemblait si terriblement aux esclaves de Michel-Ange ${ }^{24}$. Elle participe à l'équilibre global du cosmos et à une dynamique tendue vers l'Au-delà qu'illustrent les effets de miroirs des petits cartouches composant le sol et le toit de la Santa Casa qui se

23. Petrus Apianus, Liber Cosmographicus, Landshut, Weyssenberg, 1524, fol. 2. Sur cette question de l'œil céleste, indispensable à la méthode et au travail du cosmographe, voir Frank Lestringant, Mapping the Renaissance World: the Geographical Imagination in the Age of Discovery, Cambridge, Polity Press, 1994.

24. Voir ici Georges Didi-Huberman, Atlas ou le gai savoir: il rapproche ces deux figures qui jouèrent un rôle important au $\mathrm{XVI}^{\mathrm{e}}$ siècle, en rappelant que l'on a longtemps pris l'esclave pour Atlas. 
réfléchissent dans les cieux et illuminent la Terre. Marie-Atlas donc, qui assure encore au milieu du XVII ${ }^{\mathrm{e}}$ siècle l'unité de l'univers en jouant si exactement son rôle de Mediatrix entre les êtres célestes et les hommes, d'intercesseur, d'instrument privilégié de la volonté du Créateur. Gumppenberg le dit sans hésiter dans l'adresse au lecteur : «au moyen des images miraculeuses, cet atlas marial enseignera avec quelle puissance la Mère de Dieu préserve le Monde. Grâce aux images miraculeuses, le lecteur comprendra que c'est Marie qui occupe la charge de cet Atlas imaginaire, car c'est elle qui retient le Ciel vengeur qui se précipite déjà sur nous $»^{25}$.

Or à lui seul, ce choix figuratif dévoile tout un pan des ambitions spécifiques de l'Atlas. Celui-ci prend résolument position dans les débats scientifiques contemporains. Tout d'abord parce qu'il réveille, dans le contexte des débats de la fin du $\mathrm{XVI}^{\mathrm{e}}$ siècle et surtout des premières décennies du $\mathrm{XVII}^{\mathrm{e}}$ siècle autour de l'héliocentrisme puis des propositions de Galilée, certaines des anciennes aspirations de la cosmographie de la Renaissance, qui entendaient penser d'un seul tenant les cieux et la Terre, unis dans un système complexe de correspondance, et surtout ne pas séparer sciences de la nature et enjeux théologiques, raison et foi. Unité de la Création et ordre providentiel fondaient cette conception spécifique de la cosmographie et des objectifs qu'elle s'assignait. L'Atlas, en ce sens, n'est pas séparable d'autres titres, plus ou moins contemporains, qui entendent souligner à travers la description d'un cosmos unifié l'action de la Providence de Dieu dans un cadre ptolémaïque très conventionnel, comme le Teatro del Cielo et della Terra de Giuseppe Rosaccio (1598) ou la Divine Cosmographie de William Hodson $(1640)^{26}$.

Au-delà, à sa manière, l'Atlas paraît poursuivre également certaines tentatives de la fin du Moyen Âge et du XVI ${ }^{\mathrm{e}}$ siècle pour harmoniser les données de l'expérience toujours plus nombreuses que fournissaient la géographie, l' astronomie et le récit biblique de la création du monde, quitte à déplacer le terrain de cette confrontation. C'est ainsi qu'il confère, par exemple, un rôle inédit à l'histoire et à

25. Atlas Marianus, édition Jaecklin, 1658, p. [XXvi].

26. Denis E. Cosgrave, «Images of Renaissance Cosmography 1450-1650», in David Woodward (dir.), The History of Cosmography, vol. 3: Cartography in the European Renaissance 1, Chicago, The University of Chicago Press, 2007, p. $73 \mathrm{sq}$. 
la preuve historique par rapport au témoignage et à la théologie dans l'attestation des interventions miraculeuses de Marie dans le monde, ou à la sollicitation des métaphores scientifiques, empruntées à Athanase Kircher et avant lui à d'autres cosmographes importants comme John Dee, pour faire comprendre la force invisible de l'action de Dieu en la comparant au fonctionnement des «pierres magnétiques ». Pour Gumppenberg, en effet, «l'aimant transmet sa puissance à l'anneau de fer de manière à ce que l'anneau puisse la transmettre à un autre anneau et ainsi de suite, comme une chaîne. Il est certain que la force miraculeuse qui réside dans l'image de Marie vient de Marie elle-même et les vrais croyants savent par une longue expérience que cette puissance s'étend aussi aux images entrées en contact avec l'image originale ${ }^{27}$. L'extension de la puissance sacrale des images originelles à leurs copies ou leurs images de contact, l'irradiation de leur pouvoir à ce qui les approche ou leur ressemble, qui constituent un ressort spécifique du commerce pèlerin et de la multiplication des devotionalia puisque les dévots viennent toucher les statues ou la relique miraculeuse ou leur faire toucher un objet qui leur appartient (vêtement, chapeau, médaille, béquille), illustre ainsi un principe fondamental que la science découvre et décrit alors mais qu'elle ne peut ni expliquer ni illustrer de manière satisfaisante et qu'elle entoure par conséquent d'un halo persistant de mystère et de spéculations métaphysiques: les aimants et le magnétisme et avec eux la transmission d'une force sans contact direct, l'efficacité à distance, la possibilité d'animer des choses inertes par elles-mêmes et de leur insuffler une puissance qu'elles n'ont pas. Et pour le cosmographe anglais John Dee, par exemple, lui-même grand collectionneur de compas, l'aimant est bien donné par Dieu aux hommes afin que ceux-ci soient en mesure de comprendre les forces cachées des choses terrestres ${ }^{28}$. L'aimant n'est une anomalie que pour ceux qui n'en comprennent pas la cause première et l'auteur véritable.

Peut-être est-ce d'ailleurs dans cette référence à question du magnétisme qu'il faut interpréter les rayons lumineux qui

27. Atlas Marianus, édition Jaecklin, 1658, p. [XXVI] et [XXIII].

28. Alexander Statman, Ordering the World. Cosmography and the Occult Sciences in Renaissance England 1519-1576, master thesis, University of Columbia, 2009, disponible en ligne: www.columbia.edu/cu/history/resourcelibrary/Statman_thesis. 
s'échappent de la Santa Casa vers la terre, comme une figuration de l'invisible action des forces surnaturelles qui s'exercent sur le monde avec plus d'efficacité encore que toutes les lois que les sciences de la nature portent alors progressivement au jour. L'action des images miraculeuses de Marie s'exerce de manière à la fois invisible et incontestable, comme la force d'attraction des aimants. Bien des images liées au culte de la Vierge, notamment dans les sodalités mariales, reprendront cette métaphore. Enfin, et dans la même perspective, si révélatrice d'un état du savoir et des conflits qui s'y jouent dans ce moment d'éloignement progressif des formes du travail scientifique et d'éclatement de la cosmographie, l'Atlas semble constituer une tentative de christianisation de la figure d'Atlas, très différente dans ses moyens mais peut-être pas tout à fait dans ses objectifs de ce que l'on pouvait observer dans des œuvres comme le célèbre saint Christophe du Kunstmuseum de Bâle évoqué au début de ce texte ${ }^{29}$. On l'a dit, ce tableau, qui remonte aux années 1560-1570, représentait un géant, s'appuyant sur un bâton, portant sur ses épaules à la fois l'Enfant Jésus et une sphère armillaire, entretenant ainsi la confusion et peut-être l'identification entre Christophe et Atlas, mais au prix d'un simple jeu de mots sur le sens de «porter le monde» et de la mise en équivalence du monde physique et de la métaphore du Roi du Monde, une solution élégante artistiquement mais pauvre scientifiquement et qui ne pouvait satisfaire Gumppenberg et ses amis cosmographes engagés dans des combats délicats contre Galilée et ses partisans.

\section{RECHRISTIANISER LES CIEUX}

De manière révélatrice, Gumppenberg choisit donc d'écarter toute représentation à travers laquelle il manifesterait son adhésion à l'une ou l'autre des conceptions du cosmos sur lesquelles les savants du $\mathrm{XVI}^{\mathrm{e}}$ siècle et du début du XVII ${ }^{\mathrm{e}}$ siècle s'affrontaient et, du même coup, de conserver un dispositif visuel obsolète, celui de la cosmographie unifiée ou globale de la fin du Moyen Âge et de la Renaissance. Le lecteur n'aperçoit aucun homme, aucun

29. Au sujet de ce tableau, cf. notamment Stephan Kemperdick et Amelie Jensen, Kreis und Kosmos: ein restauriertes Tafelbild des 15. Jahrhunderts. Kunstmuseum Basel, 18. August-11. November 2007, Petersberg, M. Imhof, 2007. 
savant, aucun grand géographe passé et pas davantage d'allusions à leurs réalisations et à leur pensée: ni sphère armillaire, ni emboîtement des sphères aristotéliciennes, ni cartographie des constellations, ni allusion à l'héliocentrisme. À la différence d'un Mercator, d'un Andreas Argoli, d'un Blaeu ou encore d'un Johann Baptist Homann, Gumppenberg peut s'abstenir de représenter ou de faire représenter sur le frontispice de son ouvrage de grands cosmographes ou géographes du passé: Atlas lui-même, Ptolémée ou Strabon ou encore Marin de Tyr; et à l'inverse d'un Francesco Robacioli, Gumppenberg peut s'abstenir de représenter audessus du globe une carte du Purgatoire et de l'Enfer ou la sphère angélique $^{30}$. Il a tout loisir d'imaginer et de donner à voir à ses lecteurs des cieux chrétiens sans devoir, par exemple, en situer précisément les lieux principaux, représenter la lune, le soleil ou la terre, et sans avoir à signifier son adhésion à l'héliocentrisme. Il réussit ainsi à proposer un point de vue unique sur le cosmos, par-delà la distinction entre ce qui relève des savoirs humains et de l'observation directe, par l'utilisation du télescope notamment, et ce qui relève de la foi et de la connaissance indirecte des desseins de Dieu. En outre, en se donnant pour objet la présence de Marie partout dans le monde, et non dans une région ou un pays, l'Atlas rompt en partie avec les topographies sacrées qui l'avaient précédé, celles d'un Ferry de Locre ou d'un Placido Samperi ${ }^{31}$. Il rend vaine ou impuissante une enquête individuelle ou la collecte par un homme unique des renseignements nécessaires à son élaboration: l'échelle, désormais celle d'une cosmographie et plus seulement d'une topographie, change et invalide le rôle jusque-là central de l'autopsie individuelle car personne ne peut voir de ses propres yeux tous ces lieux ou visiter tous ces sanctuaires. Il faut assembler et rassembler des informations innombrables et toujours susceptibles

30. Francesco Robacioli, Teatro del cielo, a della terra, Brescia, Francesco Robacioli, 1602. Cf. aussi, sur ces motifs, Rodney W. Shirley, The Mapping of the World: Early Printed World Maps, 1472-1700, Londres, Holland Press, 1993, p. 236.

31. Ferry de Locre, Maria Augusta Virgo Deipara in septem libros tributa. Chronico et Notis ad calcem illustrata, Arras, Robert Maudhuy, 1608; Placido Samperi, Iconologia della gloriosa Vergine madre di Dio, Maria, protettrice di Messina, divisa in cinque Libri, ove si ragiona delle imagini di Nostra Signora, che si riveriscono ne' tempii e cappelle più famose della città di Messina, Messine, Giacomo Matthei, 1644, exemplaire BnF K 268. 
de nouveaux ajouts, ce qui est le propre de l'Atlas comme forme savante justement. C'est un ouvrage sans borne qu'il réalise, un univers savant en expansion indéfinie puisque de nouvelles images pourront plus tard y prendre place, y trouver leur place exacte dans un classement qui ne laisse rien au hasard. La question de la validation des miracles est ainsi réglée puisqu'il ne peut plus être question d'enregistrer, comme dans les vieux Mirakelbücher, des listes sans fin de témoignages isolés et fragiles, qui n'étaient que des vues subjectives et au fond des opinions, insuffisantes pour décrire ou sentir des vérités et des faits surnaturels. Du coup, pour réfuter les critiques des «hérétiques» qui ne reconnaissent pas les témoignages du peuple, Gumppenberg dit avoir «résolu de faire appel à des témoins divers, mais sans me contenter d'en citer deux ou trois, bien que cela suffise normalement comme preuve. Non, c'est tout un peuple qui me servira de témoin. Et si cela ne suffit pas, je veux faire appel à des nations et à des pays entiers $»^{32}$. L'Atlas rassemblera donc des faits, attestés, vérifiés, situés et garantis par des informateurs dignes de foi, et non des opinions et des témoignages humains, toujours subjectifs. Ces faits, amassés et confirmés par des centaines de régents, de provinciaux, de théologiens, recoupés et mis en récit cohérent par l'auteur sont au fond l'exact pendant du travail des auteurs de globes et de mappemondes qui reliaient des informations qui leur avaient été communiquées par des voyageurs, des polygraphes, des géographes. La globalisation du savoir passe donc par le travail collectif et la désingularisation. Certes, les faits en question sont ici d'un autre ordre que dans le travail géographique et ils ne peuvent être saisis par les mêmes facultés humaines; ils ne concernent pas les lois de la nature et ne peuvent fonder un savoir exclusivement humain ${ }^{33}$. Mais le principe reste bien le même: celui de la construction d'un savoir global et objectivé par la disparition du point de vue ${ }^{34}$.

32. Adresse au lecteur, Atlas Marianus, édition Jaecklin, 1658, p. [xxIv] sq.

33. Denis E. Cosgrave, «Images of Renaissance Cosmography 1450-1650», art. cit., p. 67: «The scale of globe and the cosmographer's role as recorder of the other's discoveries denied the possibility of personnal autopsy».

34. Adresse au lecteur, Atlas Marianus, édition Jaecklin, 1658, p. [xxv] sq. : «Je conclurai avec les paroles du Christ: "Allez, et annoncez ce que vous avez vous-même vu et entendu. Les aveugles voient, les paralytiques marchent, les exclus sont purifiés, etc." Celui qui, contre ces évidences et contre lui-même, veut jouer au plus malin, celui-là le fera à ses dépens». 
Tout indique donc que l'Atlas prend place, ou plus exactement position, dans les combats de la science jésuite du XVII ${ }^{\mathrm{e}}$ siècle et qu'il doit être rapproché de ces autres entreprises. On pense notamment à celles qui continuent, comme le fait par exemple Giovanni Battista Riccioli en 1651, à défendre le système de Tycho Brahe contre les théories de Kepler et de Galilée, dans des ouvrages qui semblent connaître un certain retentissement dans le prolongement des décrets de Paul V (1616) et Urbain VIII (1633) contre l'héliocentrisme copernicien ${ }^{35}$. L'Atlas et la place qu'il confère à Marie dans l'équilibre de l'univers sont l'une des tentatives de christianisation des constellations qui se multiplient autour de la Compagnie de Jésus et de sa sphère d'influence, sous différentes formes. En 1627, par exemple, Julius Schiller, un juriste et cartographe d'Augsbourg, publie sous le titre explicite de Coelum Stellatum Christianum un atlas de cartes célestes dans lequel les constellations désignées par les signes du zodiaque et des dieux antiques ont été remplacées par des constellations chrétiennes $^{36}$ : comme Gumppenberg a plus tard remplacé Atlas par Marie, les constellations du zodiaque sont renommées avec les noms des apôtres, les cieux septentrionaux sont identifiés au Nouveau Testament et les cieux méridionaux à l'Ancien Testament. Abraham et Isaac se substituent ainsi au Centaure, saint André au Taureau, Marie-Madeleine à Cassiopée... ${ }^{37}$. Pour l'assister dans cette entreprise qui conjugue une ambition scientifique élevée et un projet religieux très clair, Schiller recourt aux conseils de membres alors célèbres de la Compagnie de Jésus comme les astronomes Johann Baptist Cysat et Paul Guldin, ou encore le philologue et historien Matthäus Rader. Il sollicite aussi la contribution du cosmographe Wilhelm Schickard, du peintre d'histoire Johann Mathias Kager et du graveur Lucas Kilian,

35. Giovanni Batista Riccioli, Almagestum novum astronomiam veterem novamque complectens observationibus aliorum et propriis novisque theorematibus, problematibus, ac tabulis promotam, 2 vol., Bologne, V. Benatius, 1651.

36. Julius Schiller, Coelum Stellatum Christianum, Augsburg, Andreas Aperger, 1627 (disponible en ligne: http://lhldigital.lindahall.org/cdm/ref/collection/astro_ atlas/id/1142).

37. Cf. au sujet de ces motifs Peter Whitfield, The Mapping of the Heavens, Londres, British Library, 1995, p. 89 sq. Voir aussi Deborah Jean Warner, The Sky Explored: Celestial Cartography, 1500-1800, New York, A. R. Liss, 1979, p. 229-230. 
qui réalise les images des nouvelles constellations - et se trouve être le beau-frère de Wolgang Kilian, qui a gravé le frontispice des premières éditions de l'Atlas Marianus. Peu importe alors que le système de Schiller n'arrive pas à détrôner les anciennes désignations des constellations: le projet de christianiser les cieux ne disparaît pas avec lui, comme le montrent deux expériences postérieures qui s'en inspirent fortement. En 1655, Wilhelm Schickard réalise ainsi une carte céleste qui ajoute des légendes chrétiennes pour suggérer des équivalents bibliques aux figures traditionnelles: Persée est comparé à David, Draco au dragon de la Révélation... En 1660 encore, Andreas Cellarius publie les cartes spectaculaires d'un Ciel entièrement christianisé dans son Harmonia Macrocosmica ${ }^{38}$.

On objectera que l'Atlas ne propose nullement une cartographie des cieux, qu'il ne renomme pas les constellations et qu'au fond il reste d'une grande discrétion sur les querelles astronomiques de la première moitié du siècle. Mais le bandeau qui, dans la voûte céleste, surmonte la tête de la Vierge à la droite de la représentation de la lune invite à nuancer cette impression. Il s'agit bien entendu d'une nouvelle substitution: six images de la Vierge remplacent la bande zodiacale traditionnelle, telle qu'elle apparaissait notamment sur l'Atlas Farnèse et dans de nombreuses autres images contemporaines, comme la représentation du système de Ptolémée dans l'Atlas de Cellarius en 1629 ou encore la gravure du roi Atlas dans le premier traité de cosmographie publié en anglais, le Cosmographical Glasse de William Cunningham (1559). Cette substitution des images de Marie, Reine des Cieux chrétiens, aux anciennes constellations de la mythologie n'a rien d'un jeu, érudit mais sans enjeu, ou d'une tentative isolée de Gumppenberg, qui ne dévoilerait rien des manières concurrentes de penser le cosmos et les puissances qui en assuraient le fonctionnement. Elle irrigue, au contraire, une partie importante de la littérature dévotionnelle du XVII ${ }^{\mathrm{e}}$ siècle et nourrit ces topographies sacrées dont l'Atlas fut sans doute l'exemple le plus célèbre.

38. Andreas Cellarius, Harmonia Macrocosmia, Amsterdam, Johann Jansson, 1660. Éd. des planches et commentaires par Robert H. van Gent, Cologne, Taschen Verlag, 2012. Reproduction des planches disponible en ligne: http://www.staff. science.uu.nl/ gent0113/cellarius/cellarius_plates.htm 
D'un côté, paraissent de nouvelles topographies sacrées consacrées à Marie, souvent sous la plume de membres de la Compagnie, en Italie, en Allemagne ou au Mexique, qui portent le nom à la fois étonnant et révélateur de zodiaque de la Vierge: c'est le cas du Zodiaco Mariano de Francisco de Florencia ${ }^{39}$, du Zodiaco di Maria du dominicain Serafino Montorio $(1715)^{40}$ ou encore du Zodiacus Rosario Marianus du jésuite Peter Paul Rosenberger $(1698)^{41}$. Ce choix lexical et polémique devait sembler d'autant plus efficace que, d'un autre côté, d'innombrables traités de dévotion mariale, de manuels de prière ou de sermons multipliaient les parallèles ou les substitutions entre litanies de la Vierge, rosaire et zodiaque, cherchant par là à la fois à conforter la dévotion au rosaire qui avait reçu une impulsion nouvelle de Pie $\mathrm{V}$ au lendemain de Lépante et à faire, justement, de celle-ci une arme de reconquête spirituelle et intellectuelle, un levier pour repenser le monde et y rétablir le surnaturel dans tous ces droits. Dans son Rosario della Sacratissima Vergine Maria, Louis de Grenade compare ainsi, dans sa traduction italienne, la dévotion au rosaire à «uno spirituale zodiaco», qui fait progressivement passer le croyant d'une étape à une autre ${ }^{42}$. En 1676, le père Bernardo

39. Francisco de Florencia, Zodiaco Mariano, en que el Sol de Justicia Christo con la salud en las alas visita como singos, y casas proprias para beneficio de los hombres los templos, y lugares dedicados a los cultos de su SS Madre por medio de la mas celebres y milagros imagines, México, Antiguo Colegio de San Ildefonso, 1755. L'ouvrage a été rédigé vers 1680, mais ne fut publié qu'au milieu du XVIII ${ }^{\mathrm{e}}$ siècle: cf. Zodiaco mariano. 250 años de la declaración pontificia de María de Guadalupe como patrona de México, México, Museo de la Basílica de Guadalupe \& Museo Soumaya, 2004.

40. Serafino Montorio, Zodiaco di Maria, ovvero le dodici provincie del Regno di Napoli, come tanti segni, illustrate da questo Sole per mezzo delle sue prodigiosissime Immagini, Naples, Paolo Severini, 1715. Les douze signes du zodiaque représentent chez Montorio les provinces du royaume de Naples; chaque étoile correspond à une image de Marie ou au lieu qui l'abrite. Voir Elisabetta Ciancio, «Le Leggende, l'iconografia, i santuari, i miracoli e gli ex voto nello Zodiaco di Mari di Serafino Montorio », La Capitanata, années XXVI-XXXI, $\mathrm{n}^{\circ} 1$, p. 183-232.

41. Peter Paul Rosenberger, Zodiacus Rosario-Marianus, dass ist Marianische Lobreden auf 12 Monath..., Augsbourg et Dillingen, Johann Caspar Bencards, 1698.

42. Louis de Grenade, Rosaria della Sacratissima Vergine Maria, Venise, Bernardo Giunti, 1587, p. 4v. L'expression se retrouve chez Juan Sagastizaval, Exortación a la santa devocion del Rosario de la Madre de Dios, dirigada a Don Phelippe III. Nuestro senor, Saragosse, Lorenzo de Robles, 1597, cf. la dédicace, p. [XI]. 
Finetti se montre plus explicite encore dans ses Riflessi di spirito e considerazioni morali sopra li Evangeli e festivita della Beatissima Vergine Maria: selon lui, «le ventre de Marie est semblable à la face du zodiaque, que le soleil parcourt» car Marie est « un signe universel», « un zodiaque céleste » ${ }^{43}$. En 1698 encore, la traduction italienne des sermons du rosaire du jésuite António Vieira, parue sous le titre La Rosa mystica ${ }^{44}$, rappelle qu'à l'instar «du zodiaque du soleil naturel qui se compose de 12 signes, le Soleil divin a un autre Zodiaque, qui se divise en 15 signes, qui sont les 15 mystères du Rosaire». Il existe donc à côté des constellations «naturelles» du zodiaque un autre cosmos, dont les mystères ne peuvent être élucidés par la seule observation.

Certains des traités publiés par des membres de la Compagnie de Jésus au cours du $\mathrm{XVII}^{\mathrm{e}}$ siècle et qui mêlent délibérément, comme l'Atlas de Gumppenberg, préoccupations scientifiques, controverses astronomiques, ambitions dévotionnelles autour du culte des saints et de la Vierge, vont au-delà encore : ils comportent eux aussi des frontispices ou des gravures qui rejoignent les choix figuratifs des éditions des années 1650 de l'Atlas. Il ne s'agit pas là de choix esthétiques destinés au seul plaisir des yeux, de décors plaisants pour attirer le chaland, mais bien de «vues de l'esprit» destinées à faire voir une conception du cosmos bien particulière. On le voit avec le Zodiacus Rosario-Marianus qui s'ouvre sur une gravure représentant une Vierge du Rosaire, avec l'Enfant dans ses bras, entouré d'un cercle dans lequel figurent tous les signes du zodiaque, accompagné chacun d'une référence biblique associée aux litanies de la Vierge. En bas, dans un cartouche, l'inscription «Spes mea in Deo» confirme cette christianisation mariale des cieux et vaut sans doute condamnation de tout usage profane des signes zodiacaux, de toute inclination vers l'occultisme, par exemple dans l'astrologie judiciaire (fig. 7). Même si le propos en est différent, le frontispice du Rosa ursina sive Sol, de Christoph

43. Bernardo Finetti, Riflessi di spirito e considerazioni morali sopra li Evangeli e festivita della Beatissima Vergine Maria, Venise, 1676, p. 138 sqq. (disponible en ligne: http://reader.digitale-sammlungen.de/de/fs 1/object/display/ bsb10262560_00005.html).

44. António Vieria, La Rosa mystica: sermoni in lode di Nostra Signora del Rosario, trad. du portugais par Giovanni Antonio Astori, Venise, Alvise Pavino, 1698, p. 66. 
Scheiner ${ }^{45}$, qui s'affronte alors durement à Galilée, mérite lui aussi d'être évoqué: il entend concilier auctoritas sacra et ratio, auctoritas profane et sensus, pour défendre sa position concernant les taches solaires et continuer à maintenir l'idée d'un système géocentrique. Là encore, les signes du zodiaque sont intégrés dans une image complexe qui subordonne les outils des sciences et de la philosophie de la nature - le calcul, l'observation, l'usage de la raison - aux dispositions, aux manifestations et aux révélations que Dieu a bien voulu donner aux hommes pour qu'ils entrevoient l'ordre du cosmos.

\section{Conserver l'Univers}

C'est dans ce contexte scientifique et religieux spécifique que s'inscrit l'Atlas avec l'ambition de faire servir à la gloire de la Reine des Cieux les nouveaux outils, notamment graphiques, du savoir astronomique et géographique que les hommes inventaient, mais aussi de célébrer les princes qui, sur terre, savaient honorer celle-ci. On peut en prendre pour preuve la transformation complète de la page de titre de l'Atlas dans la traduction allemande parue à Prague en 1717 (fig. 8).

Cette fois, il n'est plus question de privilégier une image unique de Marie, aussi célèbre soit-elle, ni de ne retenir qu'un seul miracle, celui du transport de la Santa Casa. C'est le miracle continué de la conservation et du fonctionnement de l'univers qu'il faut mettre sous les yeux du lecteur, pour le détourner des explications que forgeait alors la mécanique classique de Newton ou de Leibniz. Non seulement Marie n'est plus seule - elle retrouve Atlas et Hercule, dans une composition à première vue infiniment plus complexe que dans les éditions de la fin des années 1650 - mais l'ensemble des éléments évoqués jusqu'ici - les sphères céleste et terrestre, la présence du Père Éternel et en fait de La Trinité, la bande zodiacale, la comparaison des souverains au Titan et au demi-dieu de la mythologie - sont enfin agencés dans une image unique. Au plus haut du Ciel, La Trinité veille sur l'univers, qui prend la forme

45. Christoph Scheiner, Rosa ursina sive sol, Bracciano, Andrea Fei, 1626-1630 (planches consultables en ligne: http://gallica.bnf.fr/ark:/12148/btv1b2600028c). 
de deux sphères superposées et supportées, difficilement, par Atlas et Hercule: le premier, à genoux, soutient la voûte céleste où l'on aperçoit nettement la bande zodiacale. C'est d'elle que surgissent les personnes divines. Le second, Hercule, porte une autre sphère, sans doute la Terre mais au prix d'une incohérence puisqu'il est en même temps à terre. Ce frontispice veut ainsi montrer qu'à l'évidence, les deux personnages de la mythologie ne suffisent pas: les dieux et les héros de l'Antiquité - et les savoirs nouveaux qu'ils symbolisent depuis les années 1570 - n'arrivent pas à eux seuls à tenir l'univers et à en expliquer la cohérence. Si l'on veut écarter l'idée cartésienne de tourbillons de matière ou la référence à la gravité newtonienne, il faut apporter d'autres explications et convoquer d'autres forces pour ne pas tout céder à ceux qui ne veulent voir que les lois de nature à l'œuvre dans le cosmos. Au sol, les souverains chrétiens doivent ainsi apporter leur concours et soulager Hercule de son immense fardeau; dans les airs, Marie et les Anges viennent euxaussi donner à l'univers l'assise dont il a besoin, sous le regard de Dieu, créateur et seul vrai soutien du monde, devant qui, rappelle la citation de Job, «s'inclinent les appuis de l'orgeuil».

Ce double concours, politique et religieux, terrestre et céleste, et ce double rappel de la prééminence divine ne sont au fond pas sans précédents. D'un côté, Atlas ou Hercule soutenant le monde avaient été très tôt associés à l'image des souverains modernes aspirant à la monarchie universelle, soit en raison de leurs titres, par exemple impérial, soit à la faveur d'un intense prophétisme politique. Une tapisserie du XVI ${ }^{\mathrm{e}}$ siècle représente ainsi Charles Quint en Hercule vêtu de la peau du lion de Némée et portant à bout de bras la sphère des cieux; la même iconographie se retrouve jusqu'au milieu du $\mathrm{XVII}^{\mathrm{e}}$ siècle au moins. Les rois de France surent également susciter de telles images faisant d'eux les garants de l'équilibre de l'univers: la page de titre de l'Atlas de Jean Le Clerc (1631), réalisée par Léonard Gautier, représentait ainsi Louis XIII en Apollon face à Henri IV, figuré en Hercule gaulois, tenant à la main le globe terrestre; en haut, Minerve et Fama encadraient une sphère couronnée, où l'on apercevait la carte de France fleurdelisée. De l'autre côté, la littérature dévotionnelle et les ouvrages de théologie avaient aussi insisté très tôt sur le rôle de Marie, «porte du Ciel» et «étoile de la Mer» dans l'organisation et surtout la conservation de l'univers: la Vierge n'avait-elle pas porté en son sein celui qui 
l'avait créée, ou, pour le dire avec Jacques Gaultier qui accumulait les autorités à ce sujet, elle avait porté celui qui portait le monde ${ }^{46}$. Quelques années plus tard, le jésuite Giovanni Pietro Pinamonti ne dit pas autre chose lorsqu'il invite les fidèles a «benire le sue Santissime Braccia, che tante volte han portato chi sostiene con tre dita tutta la Terra $»^{47}$.

On comprend alors le rôle éminent que lui attribuent les Conférences théologiques et spirituelles sur les grandeurs de la très Sainte Vierge Marie du capucin Louis-François d'Agentan au XVIII ${ }^{\text {e }}$ siècle: "Saint Bernard dit expressement, que c'est pour l'amour de la sainte Vierge que tout le monde a été créé. Saint Fulgence tient pour assuré que c'est elle qui conserve et qui soutient le monde, et que sans sa protection il y a longtemps que le Ciel et la Terre seroient renversés $»^{48}$. Et l'on arrive même à saisir par quels détours le père jésuite Paolo Segneri arrive à démontrer que Dieu a créé l'univers pour servir également à la gloire de Marie, «Imperadrice sovrana dell'Universo»: «Frabbricava egli il Sole perché pensava che questo un di dovea formare il real manto alle Vergine col suo finissimo oro! Fabbricava egli la Luna perché pensava che questa un di dovea provvedere di reggio suolo la Vergine col suo tertissimo argento. Fabbricava egli le Stelle perché pensava che d'esse un di dovea venire la Vergine incoronata ${ }^{49}$.

Le choix d'intituler son livre Atlas et d'en donner à chaque édition une illustration figurative sophistiquée offre donc durablement à Gumppenberg, à ses éditeurs et à ses traducteurs, un moyen,

46. Jacques Gaultier, Table chronographique de l'estat du christianisme la naissance de Jésus-Christ jusques à l'année 1672, Lyon, Laurent Arnaud \& Pierre Borde, 1673, p. 420.

47. Giovanni Pietro Pinamonti, Opere del padre Gio. Pietro Pinamonti della compagnia di Gesu, con un breve ragguaglio della sua vita, Parme, Paolo Monti, all'insegna della Fede, 1706, p. 346. Propos comparables chez Giuseppe Maria Sardi, Sermoni per infervorare nella divozione verso l'augustissima regina de' cieli Maria sempre Vergine, composti e recitati in varie occasioni dal padre maestro..., Appresso Lorenzo Rivan Monti a S. Salvatore, 1742, p. 143.

48. Louis-François d'Argentan, Conférences théologiques et spirituelles sur les grandeurs de la Très Sainte Vierge Marie, Rouen, François Vaultier, 1680, p. [XIX].

49. Paolo Segneri, Opere del padre Paolo Segneri della Compagnia di Gesù, distribuite in quattro tomi Con un breve ragguaglio della di lui vita : Quaresimale del padre Paolo Segneri della Compagnia di Gesu', vol. 2, Venise, Baglioni, 1738, p. 367. 
détourné mais finalement efficace, de prendre position dans les débats contemporains des sciences et de la philosophie de la nature. Retournant contre la science de la Renaissance puis contre la mécanique classique l'une des figures qui en avait symbolisé le renouveau et les ambitions, ils réussissent à faire de cette immense somme d'histoires, de récits, d'anecdotes plus ou moins solides l'un des lieux où observer au plus près certaines des stratégies déployées dans les réseaux des savants de la Compagnie, qui entendent à la fois être des acteurs de la «révolution scientifique» et refuser «l'autonomisation de la science $»^{50}$.

olivier.christin@unine.ch

50. Je remercie Estelle Leutrat, Fabrice Flückiger et Anton Serdeczny de leur aide dans la préparation et la rédaction de ce texte. Il est en partie aussi le leur. 




Fig. 1: Gerhard Mercator, Atlas sive Cosmographicae Meditationes de Fabrica Mundi et Fabricati Figura, Duisbourg, Albert Buys, 1595.

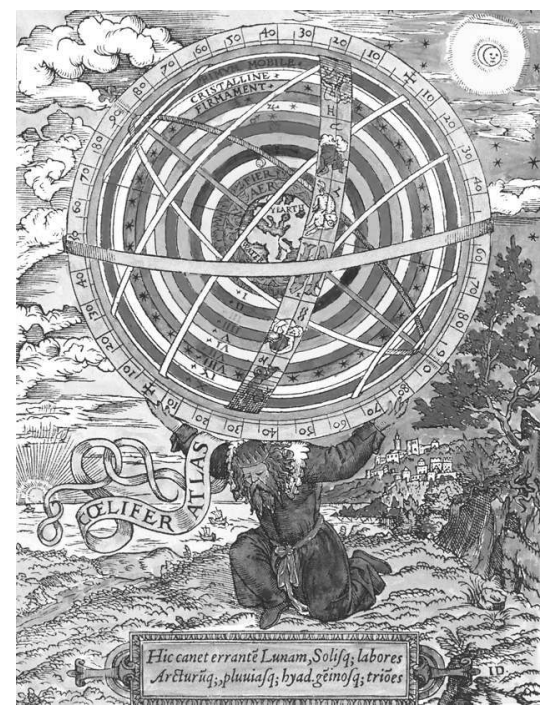

Fig. 2: William Cunningham, The Cosmographical Glasse, conteinyng the Pleasant Principles of Cosmographie, Geographie, Hydrographie or Navigation, Londres, John Day, 1559. 


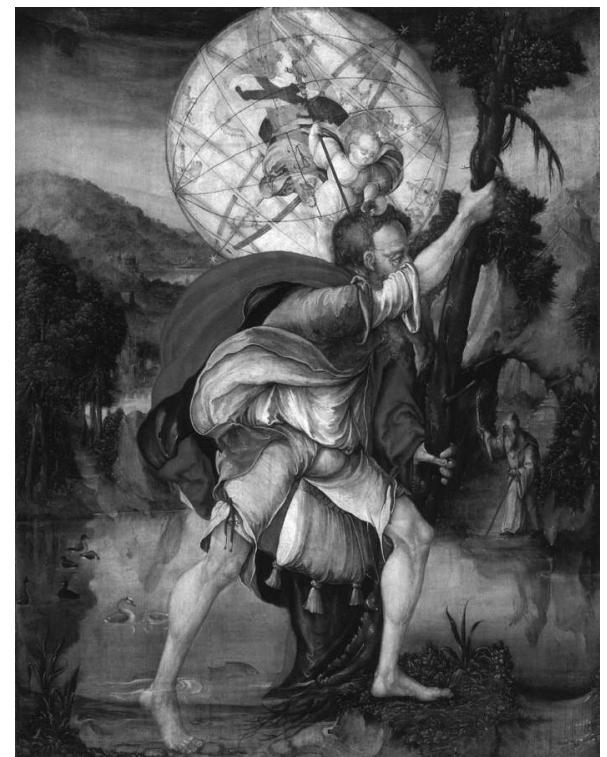

Fig. 3 : Meister von Messkirch, Saint Christophe, vers 1560, Kunstmuseum Basel.

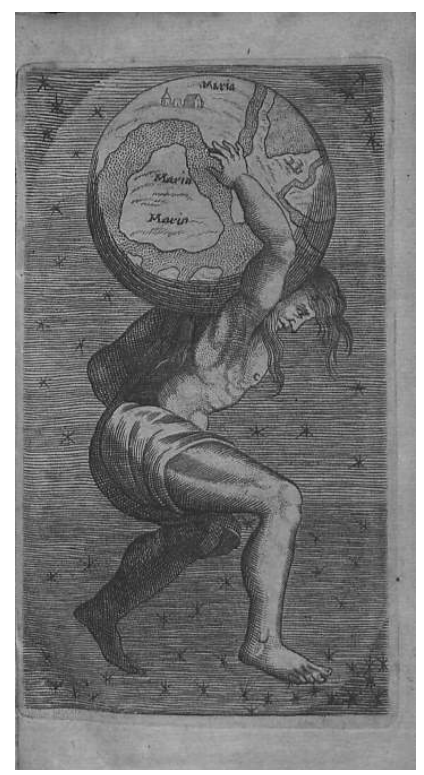

Fig. 4: Antonín Frozín, Obrowisstě Maryánského Atlanta, Prague: Jiř́i Laboun, 1704 : traduction et adaptation de l'Atlas Marianus de Wilhelm Gumppenberg. 


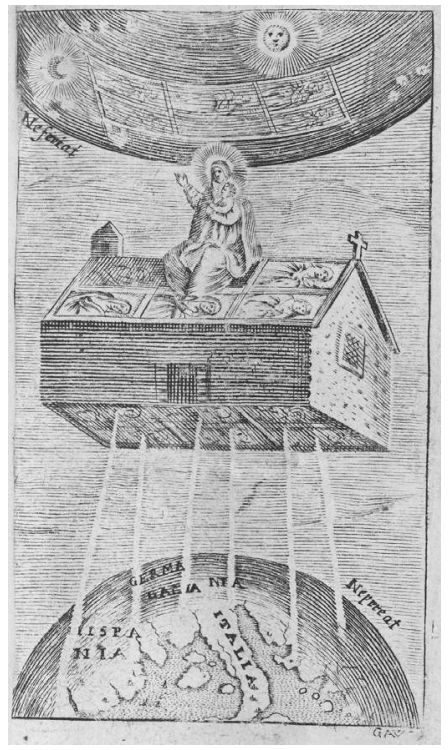

Fig. 5 : Wilhelm Gumppenberg, Marianischer Atlas : das ist wunderthätige Mariabilder so in aller christlichen Welt mit Wunderzaichen berhuembt, Munich, Johann Jaecklin, 1658, tome I.

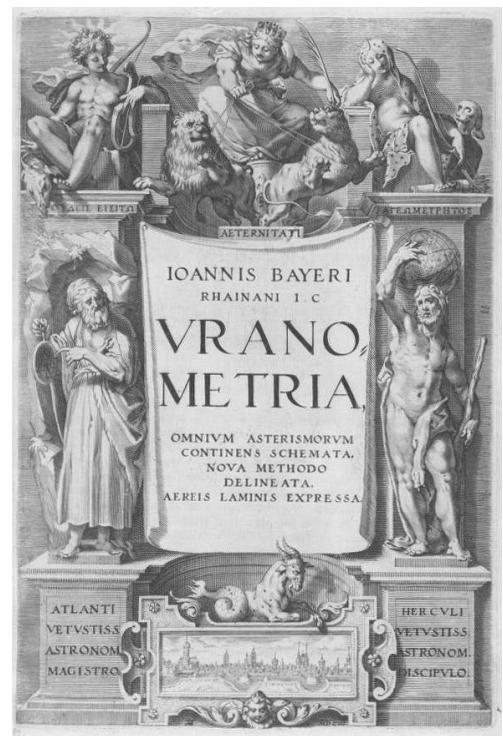

Fig. 6: Johann Bayer, Uranometria, omnium asterismorum continens schemata, nova methodo delineata, aeris laminis expressa, Augsbourg, C. Mangus, 1603. 


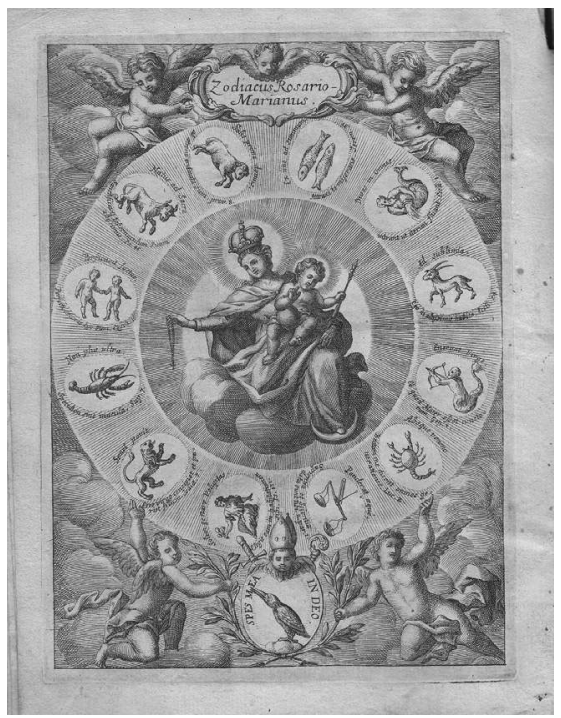

Fig. 7: Peter Paul Rosenberger, Zodiacus Rosario-Marianus, dass ist Marianische Lobreden auf 12 Monath..., Augsbourg et Dillingen, Johann Caspar Bencard, 1698.

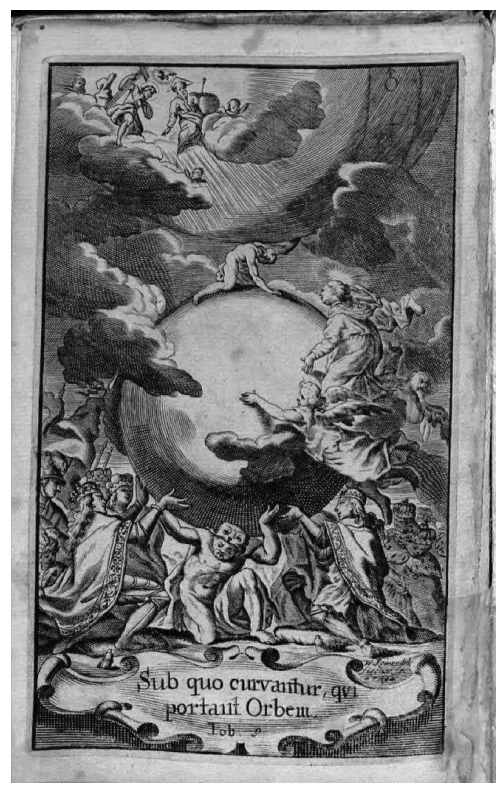

Fig. 8: Wilhelm Gumppenberg, Marianischer Atlas, oder Beschreibung der Marianischen Gnaden-Bilder durch die gantze Christen-Welt, Prague, Barbara Beringer, 1717. 